José Mário Nunes da Silva a

Lúcia de Fátima Almeida de Deus Moura a

a Universidade Federal do Piauí, Programa de Pós-Graduação em Ciências e Saúde. Teresina, PI, Brasil.

Contato:

José Mário Nunes da Silva

E-mail:

zemariu@hotmail.com

Este trabalho é baseado na dissertação de mestrado de José Mário Nunes da Silva intitulada "Capacidade para o trabalho de cirurgiões-dentistas da Estratégia Saúde da Família", defendida em 2014 no Centro de Ciências e Saúde da Universidade Federal do Piauí.

O trabalho não foi apresentado em reunião científica.

Os autores declaram não haver conflitos de interesse e que o trabalho não foi subvencionado.

Recebido: 30/07/2015

Revisado: 18/07/2016

Aprovado: 18/07/2016

\section{Capacidade para o trabalho de cirurgióes-dentistas da atenção básica: prevalência e fatores associados}

\author{
Work ability of primary care dentists: \\ prevalence and associated factors
}

\section{Resumo}

Objetivo: avaliar os fatores associados à capacidade para o trabalho de cirurgiões-dentistas. Métodos: a amostra foi constituída por 167 cirurgiões-dentistas vinculados à Estratégia Saúde da Família. Foram aplicados questionários sobre a capacidade para o trabalho, dados sociodemográficos, caraterísticas relacionadas ao trabalho, aspectos de saúde autorreferidos, estilo de vida e fadiga. $\mathrm{Na}$ análise univariada utilizou-se a estatística descritiva; na bivariada, o teste Qui-quadrado de Pearson, e, na multivariada, a regressão de Poisson. Resultados: $46,7 \%$ da amostra mostrou capacidade inadequada para o trabalho, sendo as variáveis independentes associadas: sexo feminino ( $R P=1,12$; IC95\%: 1,01-1,23), limpeza não adequada ( $R P=1,14$; IC95\%: 1,01-1,29), ambiente úmido ( $\mathrm{RP}=1,35$; IC95\%: 1,15-1,59), ambiente de trabalho com muito barulho $(\mathrm{RP}=1,10$; IC95\%: 1,01-1,30), tarefas repetitivas e monótonas $(\mathrm{RP}=1,36$; IC95\%: 1,20-1,55), insatisfação com sono (RP=1,15; IC95\%: 1,05-1,25), estado de saúde regular $(\mathrm{RP}=1,25$; IC95\%: 1,10-1,41), apresentar de uma a quatro morbidades diagnosticadas ( $\mathrm{RP}=1,14$; IC95\%: $1,04-1,26)$, cinco ou mais morbidades $(\mathrm{RP}=1,52$; IC95\%: 1,38-1,70) e níveis altos de percepção de fadiga $(\mathrm{RP}=1,15$; IC95\%: 1,04-1,26). Conclusão: A amostra estudada apresentou incapacidade para o trabalho, que foi associada a fatores sociodemográficos, características do trabalho, aspectos de saúde e altos níveis de fadiga.

Palavras-chave: saúde do trabalhador; fadiga/epidemiologia; odontólogos; condições de trabalho; estudos transversais.

\begin{abstract}
Objective: to evaluate the factors associated with the dentists' ability to work. Methods: the sample consisted of 167 dentists' linked to the Estratégia Saúde da Família [Family Health Strategy]. We handed out questionnaires about work ability, sociodemographic data, work-related characteristics, self-reported health aspects, lifestyle, and fatigue. We used descriptive statistics in univariate analysis; Pearson's chi-squared test in the bivariate, and, Poisson regression in the multivariate. Results: $46.7 \%$ of the sample showed inadequate work ability, with the following associated independent variables: women ( $P R=1.12$; 95\%CI: 1.01-1.23), inadequate cleaning ( $P R=1.14 ; 95 \% C I$ : 1.01-1.29), humid environment ( $P R=1.35 ; 95 \% C I$ : 1.15-1.59), very noisy work environment ( $P R$ =1.10; 95\%CI: 1.01-1.30), repetitive and monotonous tasks ( $P R=1.36 ; 95 \% C I$ : 1.20-1.55), dissatisfaction with sleeping ( $P R=1.15$; 95\%CI: 1.05-1.25), regular health state $(P R=1.25 ; 95 \% C I: 1.10-1.41)$, having from one to four diagnosed morbidities ( $P R=1.14 ; 95 \% C I$ : 1.04-1.26), five or more morbidities ( $P R=1.52$; 95\%CI: 1.38-1.70), and high levels of fatigue perception $(P R=1.15 ; 95 \% C I$ : 1.04-1.26). Conclusion: The studied sample showed incapacity to work, which was associated with demographic factors, work characteristics, health aspects , and high levels of fatigue.
\end{abstract}

Keywords: occupational health; fatigue/epidemiology; dentists; working conditions; cross-sectional studies. 


\section{Introdução}

Estudos têm demonstrado como diferentes tipos de ocupações podem afetar a saúde de trabalhadores $^{1,2}$. O exercício da odontologia enquadra-se nas categorias profissionais que mais sujeitam os seus praticantes a se exporem a múltiplos fatores de risco para o desenvolvimento de distúrbios osteomusculares relacionados ao trabalho (DORT). Dentre esses fatores, destaca-se a manutenção prolongada de posturas estáticas, atividades repetitivas, longas jornadas de trabalho, ambiente de trabalho mal projetado, estresse e manejo de materiais químico e biológico ${ }^{3-5}$.

A prevalência de distúrbios musculoesquelético em cirurgiões-dentistas (CDs) varia de $61 \%$ a $96,9 \%{ }^{6-}$ ${ }^{8}$, fato que provoca elevados índices de absentismo, incapacidade temporária ou permanente para o trabalho com reflexos negativos na qualidade dos serviços prestados à população e na capacidade para $o$ trabalho desses profissionais ${ }^{1,5,8}$.

A Capacidade para o trabalho é a resultante das condições de saúde do trabalhador - inclusive suas capacidades físicas e mentais - em relação às demandas físicas, mentais e sociais que o trabalho apresenta, podendo ser mantida ou restaurada, se medidas preventivas e terapêuticas forem tomadas em relação ao trabalhador 9,10. Dessa forma, diferentes categorias profissionais têm sido pesquisadas quanto à sua capacidade para o trabalho no decorrer dos anos, por exemplo, trabalhadores de indústrias têxteis com diferentes turnos ${ }^{11}$, trabalhadores de enfermagem ${ }^{10,12}$, trabalhadores da área administrativa $^{13}$, eletricitários ${ }^{14}$, motoristas de ônibus ${ }^{15}$, bombei$\operatorname{ros}^{16}$, professores ${ }^{17}$ e trabalhadores em geral ${ }^{18}$.

Entretanto, no que concerne aos cirurgiões-dentistas, os estudos atuais têm sido focados principalmente em disfunções ergonômicas ou de biossegurança ${ }^{1,8,19}$, não sendo detectadas - seja na literatura nacional ou internacional- pesquisas que analisem a capacidade para o trabalho e sua associação com aspectos organizacionais nessa categoria profissional. Assim, esta pesquisa tem por objetivo avaliar os fatores associados à capacidade para o trabalho de CDs que trabalham na Estratégia Saúde da Família (ESF).

\section{Métodos}

Foi realizado um estudo epidemiológico de corte transversal. Fizeram parte da pesquisa cirurgiões-dentistas vinculados à ESF no município de Teresina, Piauí, no período de janeiro a março de 2014.

Como critério de inclusão no estudo, os CDs participantes deveriam estar em exercício profissional no período da coleta de dados e aceitar participar da pesquisa. Os CDs que não estivessem realizando atividades assistenciais diretas (trabalhando em atividade administrativa ou afastados do trabalho por qualquer motivo) foram excluídos da pesquisa.

A amostra foi do tipo probabilística aleatória simples, considerando um total de 220 CDs na ESF trabalhando no período pesquisado. O cálculo da amostra foi estimado mediante uma prevalência de 50\% para as variáveis relacionadas ao desfecho, margem de erro de $5 \%$ para um intervalo de confiança de $95 \%$, efeito do desenho de $1,0^{20}$, totalizando uma amostra ideal para o desenvolvimento do estudo de 140 CDs. A amostra foi aumentada em $20 \%$, pressupondo uma taxa de não resposta de $10 \%$ e mais $10 \%$ para controlar fatores de confusão, resultando em uma amostra final de 167 cirurgiões-dentistas.

Antes da aplicação dos instrumentos de coleta, foi realizado um estudo piloto com $10 \mathrm{CDs}$ não pertencentes à ESF para testar e avaliar o método proposto para o estudo; os participantes envolvidos no estudo piloto não foram incluídos como sujeitos da pesquisa no estudo principal.

O levantamento dos dados é apresentado a seguir:

A capacidade para o trabalho foi avaliada por meio do Índice de Capacidade para o trabalho (ICT) ${ }^{9}$, traduzido e validado para o Brasil ${ }^{21}$, que permite avaliar a capacidade para o trabalho a partir da percepção do próprio trabalhador, obtido a partir de dez questões sintetizadas em sete dimensões. A pontuação varia de 7 a 49 pontos.

Para a construção da variável desfecho capacidade inadequada para o trabalho, foi usado o ponto de corte sugerido por Kujala et al. ${ }^{22}$ de acordo com a idade do trabalhador. Para indivíduos com idade entre 18 e 34 anos (valor inferior a 40 pontos) e a partir de 35 anos (valor inferior a 37 pontos). O coeficiente de confiabilidade interna (alfa de Cronbach) dos itens foi 0,79.

As variáveis independentes para o estudo foram agrupadas em cinco blocos de informações:

1. Sociodemográfico: sexo; faixa etária; estado civil e renda familiar mensal.

2. Ocupacionais: local, regime e tempo de trabalho, jornada diária e semanal. No que se refere à organização do trabalho, foi aplicado um questionário autorizado e adaptado de Vasconcelos et al. ${ }^{10}$ referente à estrutura organizacional e ambiente físico do trabalho com respostas que variam de: sempre ou muito frequente, às vezes pouco frequente ou pouquíssimo frequente ou nunca.

3. Estilo de vida: prática de atividade física; consumo de álcool; fumo e estado nutricional segundo o índice de massa corporal. 
4. Aspectos de saúde autorreferidos: satisfação com sono, estresse, estado de saúde, além da quantidade de morbidades referidas com diagnóstico médico obtida através do ICT.

5. Fadiga: foi utilizado o questionário de percepção de fadiga ${ }^{23}$, adaptado e validado para o português ${ }^{11}$, composto por 30 questões de múltipla escolha com escore variando de 30 até 150 pontos, correspondendo a menor e maior fadiga respectivamente. Os escores compreendidos entre 30 e 72 foram considerados baixa fadiga, e acima de 72 , alta fadiga (corte pelo terceiro quartil). O coeficiente alfa de Cronbach foi de 0,93.

Os dados foram organizados e tabulados utilizando o Microsoft Excel versão 2010 para Windows e as análises estatísticas foram feitas por meio do SPSS versão 18.0 para Windows (SPSS Inc. Chicago, IL 60606, EUA).

Na análise univariada foram aplicados os procedimentos de estatística descritiva. Na análise bivariada para associar a capacidade inadequada para o trabalho e as variáveis sociodemográficas relacionadas ao trabalho, aspectos de saúde autorreferidos, estilo de vida e fadiga, foi utilizado o teste Qui-quadrado de Pearson $\left(\chi^{2}\right)$.

Para a construção do modelo final foi realizada uma análise multivariada por meio da regressão de Poisson com variância robusta dos erros-padrão ${ }^{24}$ com todas as covariáveis de interesse que apresentaram $\mathrm{p}<0,20$ na análise bivariada. Calcularam-se as razões de prevalência brutas e ajustadas com seus respectivos intervalos de confiança de 95\% (IC95\%), utilizando-se um modelo hierárquico no qual as variáveis de interesse foram ordenadas segundo sua precedência sobre o desfecho de interesse ${ }^{25}$ e significância obtida pelo teste de Wald para heterogeneidade. Em todas as análises realizadas foi utilizado um nível de significância de 5\%.

O projeto de pesquisa foi aprovado pelo Comitê de Ética e Pesquisa da Universidade Federal do Piauí (UFPI) com o Certificado de Apresentação para Apreciação Ética (CAAE) no 22135013.8.0000.5214. Todos os participantes da pesquisa assinaram um termo de Consentimento Livre e Esclarecido (TCLE), conforme o que preconiza a resolução $n^{\circ} 466 / 12$ do Conselho Nacional de Saúde.

\section{Resultados}

Observou-se na amostra uma amplitude de variação etária de 23,0 a 68,0 anos e que nesta a maioria dos cirurgiões-dentistas era do sexo feminino, na faixa etária de 23-39 anos, com idade média 40,3 anos $(\mathrm{dp}=10,7)$, predominantemente casado $(\mathrm{a}) /$ união estável; e com renda familiar maior que 10 salários mínimos (47\%). Quanto ao estilo de vida dos CDs, a maioria praticava algum tipo de atividade física, não era tabagista, não consumia bebida alcoólica e possuía estado nutricional eutrófico, com índice de massa corporal médio de $24,0 \mathrm{~kg} / \mathrm{m}^{2}$ $(\mathrm{dp}=3,0)$ (Tabela 1).

A Tabela 1 mostra que maioria dos CDs possuía mais de um vínculo empregatício e tempo de trabalho nas Unidades Básicas de Saúde menor que 10 anos, com média de 8,9 anos $(9,0)$. A jornada de trabalho prevalente no estudo situou-se entre 8 e 9,9 horas diárias, porém a média da amostra foi de 7,8 horas $(\mathrm{dp}=2,4)$.

A Tabela 2 apresenta aspectos relacionados ao ambiente físico e à organização do trabalho relatados pelos CDs.

Uma parte expressiva dos CDs relatou estar insatisfeita com o sono e considerou seu estado de saúde como regular, a maioria se sente estressada às vezes. A média do escore de percepção de fadiga foi de 60,8 pontos $(\mathrm{dp}=16,1)$, variando entre 32,0 e 103,0 pontos e $26,3 \%$ tiveram alto nível de fadiga (Tabela 3).

O ICT, na amostra pesquisada, teve média de 38,1 pontos ( $d p=6,3$ ), variando de 18,0 a 49,0 pontos. Após a categorização proposta por Kujala et al. ${ }^{22}$, observou-se que 53,3\% dos indivíduos foram considerados como tendo capacidade para o trabalho adequada e 46,7\% inadequada. Foram observados altos valores do ICT nas sete dimensões aferidas pelo instrumento, em proporções expressivas da amostra (Tabela 4).

Os resultados das análises bivariadas mostraram que houve associação significativa entre a incapacidade para o trabalho com o sexo dos participantes $(\mathrm{p}=0,038)$, ambiente úmido $(\mathrm{p}=0,044)$, tarefas repetitivas e monótonas $(p<0,001)$, satisfação com o sono ( $p<0,001)$, estresse autorreferido $(p=0,029)$, estado de saúde autorreferido $(p=0,005)$, morbidades por diagnóstico médico $(\mathrm{p}<0,001)$ e percepção da fadiga $(\mathrm{p}<0,001)$.

As variáveis que não obtiveram significância estatística na análise bivariada, mas que obtiveram um $\mathrm{p}<0,20$ e foram inseridas na análise múltipla, foram: tempo de trabalho $(p=0,080)$, limpeza adequada $(\mathrm{p}=0,181)$, sistema de refrigeração adequado ( $p=0,111)$, muito barulho no ambiente de trabalho $(\mathrm{p}=0,172)$, ambiente de trabalho com odor desagradável $(\mathrm{p}=0,081)$, ritmo de trabalho excessivo $(p=0,076)$, número de auxiliar de saúde bucal suficiente $(p=0,105)$, praticar atividade física $(p=0,056)$, consumo de bebida alcoólica $(p=0,116)$. Gênero, faixa etária e tempo de trabalho foram tomados como variáveis de controle no modelo. 
Tabela 1 Distribuição das variáveis sociodemográficas, estilo de vida e ocupacionais dos cirurgiões-dentistas da ESF de Teresina, Piauí, 2014

\begin{tabular}{|c|c|c|}
\hline Variáveis & $n$ & $\%$ \\
\hline \multicolumn{3}{|l|}{ Sociodemográficas } \\
\hline \multicolumn{3}{|l|}{ Sexo } \\
\hline Masculino & 72 & 43,1 \\
\hline Feminino & 95 & 56,9 \\
\hline \multicolumn{3}{|l|}{ Faixa etária } \\
\hline 23-39 anos & 96 & 57,5 \\
\hline$\geq 40$ anos & 71 & 42,5 \\
\hline \multicolumn{3}{|l|}{ Média $=40,3$} \\
\hline \multicolumn{3}{|l|}{ Desvio-padrão $=10,7$} \\
\hline \multicolumn{3}{|l|}{ Estado civil } \\
\hline Não casado (a) & 66 & 39,5 \\
\hline Casado (a)/União estável & 101 & 60,5 \\
\hline \multicolumn{3}{|l|}{ Renda familiar (em SM) } \\
\hline $4 \vdash 5$ & 11 & 6,6 \\
\hline $5 \vdash 10$ & 77 & 46,1 \\
\hline$\geq 10$ & 79 & 47,3 \\
\hline \multicolumn{3}{|l|}{ Estilo de vida } \\
\hline \multicolumn{3}{|l|}{ Pratica atividade física? } \\
\hline Sim & 117 & 70,1 \\
\hline Não & 50 & 29,9 \\
\hline \multicolumn{3}{|l|}{ Fuma? } \\
\hline Sim & 4 & 2,4 \\
\hline Não & 163 & 97,6 \\
\hline \multicolumn{3}{|l|}{ Consome bebida alcoólica? } \\
\hline Sim & 48 & 28,7 \\
\hline Não & 119 & 71,3 \\
\hline \multicolumn{3}{|l|}{ Estado nutricional (IMC kg/m²) } \\
\hline Eutrófico & 117 & 70,1 \\
\hline Excesso de peso & 50 & 29,9 \\
\hline \multicolumn{3}{|l|}{ Média $=24,0$} \\
\hline \multicolumn{3}{|l|}{ Desvio-padrão $=3,0$} \\
\hline \multicolumn{3}{|l|}{ Ocupacionais } \\
\hline \multicolumn{3}{|l|}{ Local de trabalho } \\
\hline Apenas serviço público (UBS) & 62 & 37,1 \\
\hline Serviço público (UBS) e privado & 105 & 62,9 \\
\hline \multicolumn{3}{|l|}{ Tempo de trabalho (UBS) } \\
\hline Até 10 anos & 114 & 68,3 \\
\hline$\geq 10$ anos & 53 & 31,7 \\
\hline \multicolumn{3}{|l|}{ Médi $a=8,9$} \\
\hline \multicolumn{3}{|l|}{ Desvio-padrão=9,0 } \\
\hline \multicolumn{3}{|l|}{ Jornada de trabalho diária (em horas) } \\
\hline $4 \vdash 8$ & 45 & 26,9 \\
\hline $8 \vdash 10$ & 105 & 62,9 \\
\hline$\geq 10$ & 17 & 10,2 \\
\hline \multicolumn{3}{|l|}{ Média=7,8 } \\
\hline Desvio-padrão $=2,4$ & & \\
\hline
\end{tabular}

Legenda: SM - salário mínimo (R\$ 720); IMC - índice de massa corporal; UBS - unidade básica de saúde; ESF Estratégia Saúde da Família. 
Tabela 2 Distribuição das características relacionadas ao ambiente físico e à organização de trabalho dos cirurgiões-dentistas da ESF de Teresina, Piauí, 2014

\begin{tabular}{|c|c|c|c|c|c|c|}
\hline \multirow[t]{2}{*}{ Variáveis investigadas* } & \multicolumn{2}{|c|}{ Sempre ou muito frequente } & \multicolumn{2}{|c|}{ Pouco frequente } & \multicolumn{2}{|c|}{$\begin{array}{l}\text { Pouquíssimo frequente } \\
\text { ou nunca }\end{array}$} \\
\hline & $n$ & $\%$ & $n$ & $\%$ & $n$ & $\%$ \\
\hline Limpeza adequada & 74 & 44,3 & 52 & 31,1 & 41 & 24,6 \\
\hline Iluminação satisfatória & 86 & 51,5 & 51 & 30,5 & 30 & 18,0 \\
\hline Sistema de refrigeração adequado & 57 & 34,1 & 61 & 36,6 & 49 & 29,3 \\
\hline Ambiente úmido & 18 & 10,8 & 52 & 31,1 & 97 & 58,1 \\
\hline Muito barulho no ambiente de trabalho & 58 & 34,7 & 54 & 32,3 & 55 & 33,0 \\
\hline Ambiente de trabalho com odor desagradável & 23 & 13,8 & 60 & 35,9 & 84 & 50,3 \\
\hline Ambiente organizado & 71 & 42,5 & 63 & 37,7 & 33 & 19,8 \\
\hline $\begin{array}{l}\text { Materiais necessários à realização dos } \\
\text { procedimentos são suficientes }\end{array}$ & 26 & 15,6 & 76 & 45,5 & 65 & 38,9 \\
\hline $\begin{array}{l}\text { Equipamentos utilizados estão adequados aos } \\
\text { procedimentos assistenciais }\end{array}$ & 46 & 27,6 & 53 & 31,7 & 68 & 40,7 \\
\hline Ritmo de trabalho excessivo & 21 & 12,6 & 84 & 50,3 & 62 & 37,1 \\
\hline Número suficiente de Auxiliares de Saúde Bucal & 140 & 83,8 & 14 & 8,4 & 13 & 7,8 \\
\hline Tarefas repetitivas e monótonas & 68 & 40,8 & 52 & 31,1 & 47 & 28,1 \\
\hline Dificuldade de comunicação entre a chefia e a equipe & 33 & 19,8 & 62 & 37,1 & 72 & 43,1 \\
\hline Chefia apoia o crescimento profissional & 41 & 24,6 & 52 & 31,1 & 74 & 44,3 \\
\hline Autonomia para tomar decisões & 72 & 43,1 & 60 & 35,9 & 35 & 21,0 \\
\hline
\end{tabular}

*Adaptado de Vasconcelos et al. ${ }^{10}$. Legenda: ESF - Estratégia Saúde da Família.

Tabela 3 Distribuição relativa aos aspectos de saúde e percepção de fadiga dos cirurgiões-dentistas da ESF de Teresina, Piauí, 2014

\begin{tabular}{|c|c|c|}
\hline Variáveis & $n$ & $\%$ \\
\hline \multicolumn{3}{|l|}{ Aspectos de saúde } \\
\hline \multicolumn{3}{|l|}{ Satisfação com sono } \\
\hline Muito insatisfeito (a) & 11 & 6,6 \\
\hline Insatisfeito (a) & 40 & 24,0 \\
\hline Nem insatisfeito (a)/Nem satisfeito (a) & 42 & 25,1 \\
\hline Satisfeito (a) & 67 & 40,1 \\
\hline Muito satisfeito (a) & 7 & 4,2 \\
\hline \multicolumn{3}{|l|}{ Estresse autorreferido } \\
\hline Sempre & 4 & 2,4 \\
\hline Frequentemente & 25 & 15,0 \\
\hline Às vezes & 98 & 58,6 \\
\hline Raramente & - & - \\
\hline Nunca & 40 & 24,0 \\
\hline \multicolumn{3}{|l|}{ Estado de saúde } \\
\hline Muito ruim & - & - \\
\hline Ruim & - & - \\
\hline Regular & 30 & 18,0 \\
\hline Bom & 113 & 67,6 \\
\hline Muito bom & 24 & 14,4 \\
\hline \multicolumn{3}{|l|}{ Percepção de fadiga*** } \\
\hline Baixa & 123 & 73,7 \\
\hline Alta & 44 & 26,3 \\
\hline
\end{tabular}

*Adaptado de Vasconcelos et al. ${ }^{10}$; *** Questionário de percepção de fadiga. Legenda: ESF - Estratégia Saúde da Família. 
Tabela 4 Pontuação do Índice de Capacidade para o Trabalho (ICT) segundo suas dimensões

\begin{tabular}{|c|c|c|c|c|c|c|c|c|c|c|c|c|}
\hline \multirow{2}{*}{ Dimensões } & \multirow{2}{*}{$\begin{array}{l}\text { Variação } \\
\text { do escore }\end{array}$} & \multicolumn{11}{|c|}{ Pontuação (\%) } \\
\hline & & 0 & 1 & 2 & 3 & 4 & 5 & 6 & 7 & 8 & 9 & 10 \\
\hline $\begin{array}{l}\text { Capacidade para o trabalho } \\
\text { comparada com a melhor de } \\
\text { toda vida }\end{array}$ & $0-10$ & - & - & - & - & 2,4 & 4,2 & 5,4 & 16,8 & 26,9 & 25,7 & 18,6 \\
\hline $\begin{array}{l}\text { Capacidade para o trabalho em } \\
\text { relação às exigências físicas }\end{array}$ & $0-10$ & - & - & 0,6 & 0,0 & 1,2 & 1,2 & 7,2 & 21,0 & 37,1 & 16,8 & 15,0 \\
\hline $\begin{array}{l}\text { Número de doenças atuais } \\
\text { diagnosticadas pelo médico }\end{array}$ & $1-7$ & & 22,8 & 25,1 & 16,8 & - & 15,0 & - & 20,4 & & & \\
\hline $\begin{array}{l}\text { Perda estimada para o trabalho } \\
\text { por causa de doenças }\end{array}$ & $1-6$ & & 0,0 & 2,4 & 7,2 & 25,1 & 35,9 & 29,3 & & & & \\
\hline $\begin{array}{l}\text { Faltas ao trabalho por doenças } \\
\text { no último ano ( } 12 \text { meses) }\end{array}$ & $1-5$ & & 0,0 & 3,0 & 13,2 & 38,3 & 45,5 & & & & & \\
\hline $\begin{array}{l}\text { Prognóstico próprio da } \\
\text { capacidade para o trabalho } \\
\text { daqui a } 2 \text { anos }\end{array}$ & 1,4 ou 7 & & 3,6 & & & 19,2 & & & 77,2 & & & \\
\hline Recursos mentais & $1-4$ & & 0,0 & 11,4 & 47,9 & 40,7 & & & & & & \\
\hline
\end{tabular}

A Tabela 5 apresenta o modelo final da análise múltipla hierarquizada, onde se observa que dentre as variáveis sociodemográficas relacionadas à capacidade inadequada para o trabalho; apenas a variável gênero mostrou-se associada: $\mathrm{RP}=1,12$.

Em relação às variáveis relacionadas ao trabalho (Tabela 5), os fatores independentes associados foram: limpeza não adequada $(\mathrm{RP}=1,14)$, ambiente úmido $(\mathrm{RP}=1,35)$, ambiente de trabalho com muito barulho $(\mathrm{RP}=1,10)$, tarefas repetitivas e monótonas $(\mathrm{RP}=1,36)$ e às vezes repetitivas e monótonas $(\mathrm{RP}=1,17)$.

As variáveis relativas aos aspectos de saúde (Tabela 5) que se mostraram associadas no modelo final foram: não está insatisfeito nem satisfeito com seu sono $(R P=1,13)$, está insatisfeito ou muito insatisfeito com sono $(\mathrm{RP}=1,15)$ e considera seu estado de saúde regular $(R P=1,25)$. Em relação às morbidades referidas, relatar uma a quatro doenças $(\mathrm{RP}=1,14) \mathrm{e}$ cinco ou mais $(\mathrm{RP}=1,52)$ estiveram associados à capacidade inadequada para o trabalho.

O modelo final (obtido por meio dos desvios residual e de Pearson) mostrou aderência à curva normal com variação de -2 a 2 , presença de poucos outliers e grau de liberdade próximo ao desvio de Pearson. Esses aspectos indicam bom ajuste do modelo na análise final.

\section{Discussão}

Os resultados da pesquisa mostraram que houve uma alta prevalência de capacidade inadequada para o trabalho entre a amostra estudada (46,7\%), corroborando outros estudos realizados no Brasil e no mundo com diferentes categorias profissionais ${ }^{10,12,17,18,26,27}$.
Na presente pesquisa, apenas a variável sociodemográfica sexo apresentou associação com a capacidade inadequada para o trabalho. A perda de capacidade para o trabalho em mulheres foi $12 \%$ mais prevalente que em homens. Esses resultados foram concordantes com pesquisas de outros autores ${ }^{10,28,29}$. Uma das explicações possíveis dessa relação é que, mesmo com todas as mudanças advindas da inserção das mulheres no mercado de trabalho, elas não deixaram de exercer a responsabilidade tradicional do cuidado das tarefas domésticas e filhos, acumulando múltiplos papéis e aumentando a sobrecarga de trabalho ${ }^{30,31}$.

Vale ressaltar que as cargas de trabalho impostas aos CDs, o desconforto físico, um posto de trabalho mal projetado sob o ponto de vista ergonômico e a má postura são fatores determinantes para o surgimento de doenças de origem ocupacional nesses profissionais, incomodando, e, algumas vezes, até incapacitando o seu desempenho profissional $^{1,3,4,32}$.

A limpeza não adequada do ambiente de trabalho na presente pesquisa esteve associada a um aumento de $14 \%$ na prevalência de capacidade inadequada para o trabalho. Esse achado está supostamente relacionado às inúmeras evidências de que um ambiente de trabalho limpo é essencial tanto para a prática odontológica segura para os pacientes quanto para a sanidade da equipe de saúde bucal. É notório que diversos procedimentos clínicos e cirúrgicos rotineiros dessa atividade favorecem a exposição de seus trabalhadores a uma grande variedade de micro-organismos potencialmente patogênicos presentes em fluidos corporais dos pacientes (sangue, saliva e secreções do trato respiratório alto) ${ }^{33,34}$. 
Tabela 5 Modelo final para os fatores associados à capacidade inadequada para o trabalho dos cirurgiões-dentistas da ESF de Teresina, Piauí, 2014 - estimativas de razão de prevalências obtidas mediante regressão de Poisson

\begin{tabular}{|c|c|c|c|c|c|c|c|}
\hline & \multirow{2}{*}{$n$} & \multicolumn{2}{|c|}{ ICT inadequado } & \multicolumn{2}{|c|}{ RP bruta } & \multicolumn{2}{|c|}{ RPajustada } \\
\hline & & $n$ & $\%$ & $R P$ & IC95\% & $R P$ & IC95\% \\
\hline \multicolumn{8}{|l|}{ Sociodemográficas } \\
\hline \multicolumn{8}{|l|}{ Sexo } \\
\hline Masculino & 72 & 27 & 37,5 & 1,0 & & 1,0 & \\
\hline Feminino & 95 & 51 & 53,7 & 1,12 & $1,01-1,24$ & 1,12 & $1,01-1,23$ \\
\hline \multicolumn{8}{|l|}{ Faixa etária } \\
\hline 23-39 anos & 96 & 42 & 43,8 & 1,0 & & 1,0 & \\
\hline$\geq 40$ anos & 71 & 36 & 50,7 & 1,05 & $0,95-1,16$ & 1,05 & $0,94-1,16$ \\
\hline \multicolumn{8}{|l|}{ Relacionadas ao trabalho" } \\
\hline \multicolumn{8}{|l|}{ Tempo de trabalho (UBS) } \\
\hline Até 10 anos & 114 & 48 & 42,1 & 1,0 & & 1,0 & \\
\hline$\geq 10$ anos & 53 & 30 & 56,6 & 1,10 & $0,99-1,23$ & 1,03 & $0,91-1,17$ \\
\hline \multicolumn{8}{|l|}{ Limpeza adequada } \\
\hline Sempre ou muito frequente & 74 & 34 & 45,9 & 1,0 & & 1,0 & \\
\hline Pouco frequente & 52 & 29 & 55,8 & 1,07 & $0,94-1,22$ & 1,00 & $0,87-1,16$ \\
\hline Pouquíssimo frequente ou nunca & 41 & 15 & 36,6 & 1,14 & $0,99-1,31$ & 1,14 & $1,01-1,29$ \\
\hline \multicolumn{8}{|l|}{ Ambiente úmido } \\
\hline Pouquíssimo frequente ou nunca & 97 & 48 & 49,5 & 1,0 & & 1,0 & \\
\hline Pouco frequente & 53 & 18 & 34,6 & 0,90 & $0,80-1,01$ & 0,84 & 0,74-0,94 \\
\hline Sempre ou muito frequente & 18 & 12 & 66,7 & 1,12 & $0,93-1,29$ & 1,35 & $1,15-1,59$ \\
\hline \multicolumn{8}{|l|}{ Muito barulho no ambiente de trabalho } \\
\hline Pouquíssimo frequente ou nunca & 55 & 20 & 36,4 & 1,0 & & 1,0 & \\
\hline Pouco frequente & 54 & 28 & 51,9 & 1,11 & $0,98-1,27$ & 1,09 & $0,95-1,27$ \\
\hline Sempre ou muito frequente & 58 & 30 & 51,7 & 1,11 & $0,98-1,26$ & 1,10 & $1,01-1,30$ \\
\hline \multicolumn{8}{|l|}{ Tarefas repetitivas e monótonas } \\
\hline Pouquíssimo frequente ou nunca & 47 & 10 & 21,3 & 1,0 & & 1,0 & \\
\hline Pouco frequente & 52 & 24 & 46,2 & 1,20 & $1,05-1,40$ & 1,17 & $1,02-1,35$ \\
\hline Sempre ou muito frequente & 68 & 44 & 64,7 & 1,36 & $1,21-1,53$ & 1,36 & $1,20-1,55$ \\
\hline \multicolumn{8}{|l|}{ Aspectos de saúde } \\
\hline \multicolumn{8}{|l|}{ Satisfação com sono } \\
\hline Satisfeito (a)/Muito satisfeito (a) & 74 & 19 & 25,7 & 1,0 & & 1,0 & \\
\hline Nem insatisfeito (a)/Nem satisfeito (a) & 42 & 25 & 59,5 & 1,27 & $1,23-1,43$ & 1,13 & $1,02-1,25$ \\
\hline Muito insatisfeito (a)/Insatisfeito (a) & 51 & 34 & 66,7 & 1,33 & $1,19-1,48$ & 1,15 & $1,05-1,25$ \\
\hline \multicolumn{8}{|l|}{ Estado de saúde } \\
\hline Bom/Muito bom & 137 & 57 & 41,6 & 1,0 & & 1,0 & \\
\hline Regular & 30 & 21 & 70,0 & 1,20 & $1,07-1,34$ & 1,25 & $1,10-1,41$ \\
\hline \multicolumn{8}{|l|}{ Morbidade referida com diagnóstico médico } \\
\hline Nenhuma & 34 & 03 & 8,8 & 1,0 & & 1,0 & \\
\hline 1-4 doenças & 87 & 34 & 39,1 & 1,28 & $1,14-1,43$ & 1,14 & $1,04-1,26$ \\
\hline 5 ou mais & 46 & 53,6 & 89,1 & 1,74 & $1,57-1,92$ & 1,52 & $1,38-1,70$ \\
\hline \multicolumn{8}{|l|}{ Fadiga ${ }^{* * * * *}$} \\
\hline Baixa & 123 & 38 & 30,9 & 1,0 & & 1,0 & \\
\hline Alta & 44 & 40 & 90,9 & 1,46 & $1,35-1,58$ & 1,15 & $1,04-1,26$ \\
\hline
\end{tabular}

Legenda:

RP: razão de prevalência, IC95\%: Intervalo de confiança. ESF - Estratégia Saúde da Família.

*Ajustada para variáveis sociodemográficas

***Ajustada para variáveis: sociodemográficas, relacionada ao trabalho e estilo de vida

*******justada para variáveis: sociodemográficas, relacionada ao trabalho, estilo de vida e aspectos de saúde.

Obs.: Dados em negrito representam razões de prevalência e intervalos de confiança estatisticamente significativos. 
Estudo de Bernardo et al. ${ }^{35}$ mostrou uma alta prevalência de estafilococos patogênicos no ambiente odontológico, sendo que algumas dessas bactérias se encontravam resistentes à maioria dos antibióticos utilizados. Barreto et al. ${ }^{36}$ afirmam, ainda, que aerossóis contaminados por bactérias podem transpor as barreiras fixas do ambiente odontológico, e que esses aerossóis são rotineiramente gerados pelos instrumentos de alta rotação e aparelhos ultrassônicos utilizados pelos CDs.

Um ambiente de trabalho com muito barulho foi responsável por um aumento de $10 \%$ na prevalência de perda da capacidade para o trabalho dos CDs. Essa é outra característica da organização de trabalho desses profissionais, que geralmente trabalham em um ambiente com alta tendência a ruídos, tais como os provenientes de motor de alta rotação, compressor, sugadores de saliva, além de ar condicionado fontes emissoras que, somadas, podem levar o profissional a um comprometimento da audição e de suas atividades ocupacionais ${ }^{37,38}$.

A umidade, nesta pesquisa, esteve associada a uma perda de $35 \%$ na capacidade para o trabalho, fato que corrobora os estudos de Karvala et al. ${ }^{39,40}$. Os autores demonstraram que a presença de umidade no ambiente de trabalho esteve diretamente associada a uma capacidade pobre para o trabalho, e como consequência, 13\% dos participantes relataram mudança de emprego e diminuição na qualidade de vida.

Tarefas repetitivas e monótonas estiveram associadas a um aumento de $36 \%$ na prevalência de capacidade inadequada, e tarefas às vezes repetitivas e monótonas, a um aumento de $17 \%$ na mesma capacidade. A literatura sobre a relação entre tarefas repetitivas e monótonas com comprometimento da saúde dos trabalhadores é vasta, principalmente porque são fatores de risco para o desenvolvimento de doenças ocupacionais. Logo, os resultados obtidos neste estudo mostram congruência com evidências apontadas nos demais ${ }^{1-6}$.

Quanto às variáveis relativas aos aspectos de saúde, não estar insatisfeito nem satisfeito com seu sono foi responsável por um aumento de 13\% na prevalência de capacidade inadequada, enquanto estar muito insatisfeito ou insatisfeito com o sono foi responsável por 15\% de aumento, resultados que se assemelham a outras pesquisas ${ }^{16,17,26,41}$.

Cirurgiões-dentistas que consideraram o seu estado de saúde regular tiveram capacidade para o trabalho diminuída em $25 \%$ quando comparados aos que relataram estado de saúde bom/muito bom. Os resultados da presente pesquisa são compatíveis com estudos de Vedovato e Monteiro ${ }^{17}$ e Vries et al. ${ }^{42}$, os quais relatam que a percepção geral de saúde está diretamente associada à capacidade para o trabalho.

No que tange à relação entre a presença de morbidades e capacidade para o trabalho, Gustafsson e Marklund ${ }^{18}$ verificaram que a presença de doenças está intimamente relacionada a uma baixa capacidade para o trabalho.

Observou-se, no estudo, que o relato de cinco ou mais doenças pelos CDs aumentou em $52 \%$ a prevalência de capacidade inadequada para o trabalho em relação aos que referiram não ter nenhuma doença. Vasconcelos et al. ${ }^{10}$ também encontraram essa relação, constatando que a capacidade para o trabalho foi $48 \%$ menor nos profissionais da enfermagem que relataram três ou mais doenças, quando comparados aos trabalhadores que não referiram nenhuma doença.

Outro resultado importante encontrado no estudo foi que $26,3 \%$ dos CDs possuíam níveis de fadiga elevados, e que a perda de capacidade para o trabalho foi $15 \%$ mais prevalente nesses indivíduos quando comparados aos que apresentaram níveis mais baixos; esse resultado está em consonância com estudos de Vasconcelos et al. ${ }^{10}$, que encontraram um aumento de 2,37 vezes na prevalência, e de Fisher et al. ${ }^{43}$, que também relataram essa associação com profissionais da enfermagem.

Esses resultados podem ser explicados pelo fato de a fadiga diminuir a habilidade dos indivíduos de realizar suas tarefas habituais, pois ela altera o estado de alerta e vigilância, causando danos sociais e psicológicos e comprometendo a capacidade para o trabalho ${ }^{43-45}$.

Esse é um fato que merece destaque, pois profissionais com níveis elevados de fadiga se tornam mais propensos a não realizarem suas atividades profissionais de maneira adequada, o que pode comprometer a saúde bucal da população por eles assistida. Em se tratando de um estudo transversal, os resultados aqui encontrados dizem respeito apenas ao período analisado, não sendo possível estabelecer relação de causa e efeito.

Outras duas potenciais limitações em estudos deste tipo devem ser consideradas: o chamado "efeito do trabalhador sadio" e a possibilidade de viés introduzido pelo autorrelato de algumas variáveis. Entretanto, quanto a essa última, opõe-se o argumento de que instrumentos de autoavaliação de aspectos de saúde têm sido utilizados em inquéritos populacionais por sua praticidade, alta validade e confiabilidade $^{12,17}$.

Deve ser considerada, também, a utilização do ICT como um indicador de avaliação e acompanhamento da capacidade para o trabalho desses profissionais, permitindo, assim, a identificação oportuna dos fatores que a afetam e subsidiando as medidas corretivas pertinentes. 


\section{Conclusão}

A pesquisa demonstrou que grande parte da população estudada apresentou capacidade inadequada para o trabalho, sendo os fatores independentes associados: sexo, limpeza não adequada, ambiente úmido, muito barulho no ambiente de trabalho, tarefas repetitivas e monótonas, a não satisfação com o sono, estado de saúde regular, presença de morbidade e níveis elevados de fadiga.

\section{Contribuição de autoria}

Silva, J.M.N.: participou da concepção, delineamento, análise e interpretação dos dados, da redação e da aprovação final da versão a ser publicada. Moura, L.F.A.D.: da concepção, delineamento, da redação e revisão crítica do artigo e da aprovação final da versão a ser publicada.

\section{Referências}

1. Alexandre PC, Silva IC, Souza LM, Magalhães Câmara V, Palácios M, Meyer A. Musculoskeletal disorders among Brazilian dentists. Arch Environ Occup Health. 2011;66(4):231-5.

2. Regis Filho GT, Michels G, Sell I. Lesões por esforços repetitivos/distúrbios osteomusculares relacionados ao trabalho em cirurgiões-dentistas. Rev Bras Epidemiol. 2006;9(3):346-59.

3. Shaik AR, Rao SB, Husain A, D'sa J. Work-related musculoskeletal disorders among dental surgeons: a pilot study. Contemp Clin Dent. 2011;2(4):30812.

4. Pozos Radillo BE, Tórrez López TM, Aguilera Velasco Mde L, Acosta Fernández M, González Perez GJ. Stress-associated factors in Mexican dentists. Braz Oral Res. 2008;22(3):223-8.

5. Puriene A, Aleksejuniene J, Petrauskiene J, Balciuniene I, Janulyte V. Self-reported occupational health issues among Lithuanian dentists. Ind Health. 2008;46(4):369-74.

6. Gupta D, Bhaskar DJ, Gupta KR, Karim B, Kanwar A, Jain A, et al. Use of complementary and alternative medicine for work related musculoskeletal disorders associated with job contentment in dental professionals: Indian outlook. Ethiop J Health Sci. 2014;24(2):117-24.

7. Sustová Z, Hodacová L, Kapitán M. The prevalence of musculoskeletal disorders among dentists in the Czech Republic. Acta Medica (Hradec Kralove). 2013;56(4):150-6.

8. Leggat PA, Kedjarune U, Smith DR. Occupational health problems in modern dentistry: a review. Ind Health. 2007;45(5):611-21.

9. Tuomi K, Ilmarinen J, Jahkola A, Katajarinne L, Tulkki A. Índice de capacidade para o trabalho. Tradução: Fischer FM (coord). São Carlos: Edufscar; 2005.

10. Vasconcelos SP, Fischer FM, Reis AOA, Moreno CRC. Fatores associados à capacidade para o trabalho e percepção de fadiga em trabalhadores de enfermagem da Amazônia Ocidental. Rev Bras Epidemiol. 2011;14(4):688-97.

11. Metzner RJ, Fischer FM. Fadiga e capacidade para o trabalho em turnos fixos de doze horas. Rev Saúde Pública. 2001;35(6):548-553.

12. Schmidt SG, Dichter MN, Bartholomeyczik S, Hasselhorn HM. The satisfaction with the quality of dementia care and the health, burnout and work ability of nurses: a longitudinal analysis of 50 German nursing homes. Geriatr Nurs. 2014;35(1):42-6.

13. Martinez MC; Latorre MRDO. Saúde e capacidade para o trabalho em trabalhadores da área administrativa. Rev Saúde Pública. 2006;40(5):851-8.

14. Martinez MC, Latorre MRDO. Fatores associados a capacidade para trabalho de trabalhadores do setor elétrico. Cad Saúde Pública. 2009;25(4):761-72.

15. Sampaio RF, Coelho CM, Barbosa FB, Mancini MC, Parreira VF. Work ability and stress in a bus transportation company in Belo Horizonte, Brazil. Ciênc Saúde Coletiva. 2009;14(1):287-96.

16. Airila A, Hakanen J, Punakallio A, Lusa S, Luukkonen R. Is work engagement related to work ability beyond working conditions and lifestyle factors? Int Arch Occup Environ Health. 2012;85:915-25.

17. Vedovato TG, Monteiro I. Health conditions and factors related to the work ability of teachers. Industrial Health. 2014;52:121-128.

18. Gustafsson K, Marklund S. Associations between health and combinations of sickness presence and absence. Occup Med. 2014;64:49-55.

19. Garcia LP, Blanck VLG. Prevalência de exposições ocupacionais de cirurgiões-dentistas e auxiliares de consultório dentário a material biológico. Cad Saúde Pública 2006;22(1):97-108.

20. Rao PSRS. Sampling methodologies: with applications. New York: Chapman \& Hall/CRC; 2000. 
21. Martinez MC, Latorre MRDO, Fischer FM. Validade e confiabilidade da versão brasileira do Índice de Capacidade para o Trabalho. Rev Saúde Pública. 2009;43(3):523-32.

22. Kujala V, Remes J, Ek E, Tammelin T, Laitinen J. Classification of work ability index among young employees. Occup Med (Lond). 2005;55:399-401.

23. Yoshitake H. Relations between the symptoms and the feeling of fatigue. In: Hashimoto K. et al. Methodology in human fatigue assessment. London: Taylor \& Francis; 1975.

24. Barros AJD, Hirakata VN. Alternatives for logistic regression in cross sectional studies: an empirical comparison of models that directly estimate the prevalence ratio. BMC Med Res Methodol; 2003.

25. Victora CG, Huttly SR, Fuchs SC, Olinto MTA. The role of conceptual frameworks in epidemiological analysis: a hierarchical approach. Int J Epidemiol. 1997;26:224-7.

26. Monteiro I, Chillida MS, Moreno LC. Work ability among nursing personnel in public hospitals and health centers in Campinas - Brazil. Work. 2012;41:316-19.

27. Costa AF, Puga-Leal R, Nunes IL. An exploratory study of the Work Ability Index (WAI) and its components in a group of computer workers. Work. 2011;39:357-67.

28. Costa G, Sartori S. Ageing, working hours and work ability. Ergonomics. 2007;50(11):1914-30.

29. Costa CSN, Freitas EG, Mendonça LCS, Alem MER, Coury HJCG. Capacidade para o trabalho e qualidade de vida de trabalhadores industriais. Ciênc Saúde Coletiva. 2012;17(6):1635-42.

30. Johansson G, Huang Q, Lindfors P. A life-span perspective on women's careers, health, and wellbeing. 2007;65(4):685-97.

31. Rotenberg L, Griep RH, Fischer FM, Fonseca MJM, Landsbergis P. Working at night and work ability among nursing personnel: when precarious employment makes the difference. Int Arch Occup Environ Health. 2009;82:877-85.

32. Reis WG, Scherer MDA, Carcereri DL. O trabalho do cirurgião-dentista na atenção primária à saúde: entre o prescrito e o real. Saude debate. 2015; 39(104):56-64.

33. Silva ICM, Asmus CIRF, Campos RC, Davis RH, Meyer A, Camara VM. Variação diária da exposição de mercúrio entre assistentes e estagiários em um consultório dentário. Rev Bras Odontol. 2014; 71(1):17-21.
34. Singh TS, Bello B, Mabe OD, Renton K, Jeebhay ME. Workplace determinants of endotoxin exposure in dental healthcare facilities in South Africa. Ann Occup Hyg. 2010;54(3):299-308.

35. Bernardo WlC, Boriollo MFG, Gonçalves RB, Höfling JF. Staphylococcus aureus ampicillinresistant from the odontological clinic environment. Rev Inst Med Trop S Paulo. 2005;47(1):19-24.

36. Barreto ACB, Vasconcelos CPP, Girão CMS, Rocha MMNP, Mota OML, Pereira SLS. Contaminação do ambiente odontológico por aerossóis durante atendimento clínico com uso de ultrassom. Braz J Periodontol. 2011;21(2):79-84.

37. Choosong T1, Kaimook W, Tantisarasart R, Sooksamear P, Chayaphum S, Kongkamol C, et al. Noise exposure assessment in a dental school. Saf Health Work. 2011;2(4):348-54.

38. Willershausen B. Hearing assessment in dental practitioners and other academic professionals from an urban setting. Head Face Med. 2014;10:1-7.

39. Karvala K, Uitti J, Luukkonen R, Nordman H. Quality of life of patients with asthma related to damp and moldy work environments. Scand J Work Environ Health. 2013;39(1):96-105.

40. Karvala K, Nordman H, Luukkonen R, Uitti J. Asthma related to workplace dampness and impaired work ability. Int Arch Occup Environ Health. 2014;87(1):1-11.

41. Camerino D, Conway PM, Sartori S, Campanini P, Estryn-Béhar M, van der Heijden BI, Costa G. Factors affecting work ability in day and shiftworking nurses. Chronobiol Int. 2008;25(2):425-42.

42. Vries HJ, Reneman MF, Groothoff JW, Geertzen JH, Brouwer S. Self-reported work ability and work performance in workers with chronic nonspecific musculoskeletal pain. J Occup Rehabil. 2013;23(1):1-10.

43. Fischer FM. Borges NS, Rotenberg L, Latorre MRDO, Soares NS, Rosa PLFS, et al. A (in) capacidade para o trabalho em trabalhadores de enfermagem. Rev Bras Med Trab. 2005;3(2):97-103.

44. Rosa PLFS, Fischer FM, Borges FNS, Soares NS, Rotenberg L, Landsbergis P. Percepção da duração do sono e da fadiga entre trabalhadores de enfermagem. R Enferm UERJ. 2007;15:100-6.

45. Medeiros Neto CF, Almeida GA, Ramos BC, Costa SKP, Silva HPA, Sousa MBC. Análise da percepção da fadiga, estresse e ansiedade em trabalhadores de uma indústria de calçados. J Bras Psiquiatr. 2012;61(3):133-8. 